OPEN ACCESS

Edited by:

Xin Wang,

Northwest A\&F University, China

Reviewed by:

Wenlin Bai,

Shenyang Agricultural University,

China

Zhibin Ji,

Shandong Agricultural University,

China

${ }^{*}$ Correspondence:

Jinquan $L$

lijinquan_nd@126.com

Yanjun Zhang

imauzyj@163.com

†These authors share co-first authorship

Specialty section:

This article was submitted to

Livestock Genomics,

a section of the journal

Frontiers in Genetics

Received: 10 March 2021 Accepted: 29 April 2021

Published: 11 June 2021

Citation:

Shang $F$, Wang $Y$, Ma R, Di Z, Wu Z, Hai E, Rong Y, Pan J, Liang L, Wang Z, Wang R, Liu Z, Zhao Y, Wang Z, Li J and Zhang Y (2021) Expression Profiling and Functional Analysis of Circular RNAs in Inner

Mongolian Cashmere Goat Hair Follicles. Front. Genet. 12:678825. doi: 10.3389/fgene.2021.678825

\section{Expression Profiling and Functional Analysis of Circular RNAs in Inner Mongolian Cashmere Goat Hair Follicles}

Fangzheng Shang ${ }^{1 t}$, Yu Wang ${ }^{2 t}$, Rong $\mathrm{Ma}^{3+}$, Zhengyang $\mathrm{Di}^{1}$, Zhihong $\mathrm{Wu}^{1}$, Erhan Hai', Youjun Rong1, Jianfeng Pan', Lili Liang', Zhiying Wang ${ }^{1}$, Ruijun Wang ${ }^{1}$, Zhihong Liu', Yanhong Zhao', Zhixin Wang ${ }^{1}$, Jinquan Li $^{2,3,4 *}$ and Yanjun Zhang ${ }^{1 *}$

${ }^{1}$ College of Animal Science, Inner Mongolia Agricultural University, Hohhot, China, ${ }^{2}$ Key Laboratory of Mutton Sheep Genetics and Breeding, Ministry of Agriculture, Hohhot, China, ${ }^{3}$ Key Laboratory of Animal Genetics, Breeding and Reproduction, Hohhot, China, ${ }^{4}$ Engineering Research Center for Goat Genetics and Breeding, Hohhot, China

Background: Inner Mongolian cashmere goats have hair of excellent quality and high economic value, and the skin hair follicle traits of cashmere goats have a direct and important effect on cashmere yield and quality. Circular RNA has been studied in a variety of tissues and cells.

Result: In this study, high-throughput sequencing was used to obtain the expression profiles of circular RNA (circRNA) in the hair follicles of Inner Mongolian cashmere goats at different embryonic stages $(45,55,65$, and 75 days). A total of 21,784 circRNAs were identified. At the same time, the differentially expressed circRNA in the six comparison groups formed in the four stages were: $d 75 \mathrm{vsd} 45,59$ upregulated and 33 downregulated DE circRNAs; d75vsd55, 61 upregulated and 102 downregulated DE circRNAs; d75vsd65, 32 upregulated and 33 downregulated DE circRNAs; d65vsd55, 67 upregulated and 169 downregulated DE circRNAs; d65vsd45, 96 upregulated and 63 downregulated DE circRNAs; and d55vsd45, 76 upregulated and 42 downregulated DE circRNAs. Six DE circRNA were randomly selected to verify the reliability of the sequencing results by quantitative RT-PCR. Subsequently, the circRNA corresponding host genes were analyzed by the Gene Ontology (GO) and the Kyoto Encyclopedia of Genes and Genomes (KEGG) pathway. The results showed that the biological processes related to hair follicle growth and development enriched by GO mainly included hair follicle morphogenesis and cell development, and the signaling pathways related to hair follicle development included the Notch signaling pathway and NF$\kappa B$ signaling pathway. We combined the DE circRNA of d75vsd45 with miRNA and mRNA databases (unpublished) to construct the regulatory network of circRNA-miRNAmRNA, and formed a total of 102 pairs of circRNA-miRNA and 126 pairs of miRNAmRNA interactions. The binding relationship of circRNA3236-chi-miR-27b-3p and circRNA3236-chi-miR-16b-3p was further verified by dual-luciferase reporter assays, and the results showed that circRNA3236 and chi-miR-27b-3p, and circRNA3236 and chi-miR-16b-3p have a targeted binding relationship. 
Conclusion: To summarize, we established the expression profiling of circRNA in the fetal skin hair follicles of cashmere goats, and found that the host gene of circRNA may be involved in the development of hair follicles of cashmere goats. The regulatory network of circRNA-miRNA-mRNA was constructed and preliminarily verified using DE circRNAs.

Keywords: circRNA, cashmere goat, hair follicles, expression profile, functional analysis

\section{BACKGROUND}

There are two kinds of hair follicles in the skin of cashmere goats, namely, primary hair follicles and secondary hair follicles. Primary hair follicles produce coarse hairs, and secondary hair follicles produce cashmere. The structural characteristics of the skin and hair follicles of cashmere goats are not only important correlates of their biological characteristics but also have a direct and important effect on the yield and quality of cashmere. The hair follicle is a skin accessory organ with complex morphology and structure; it controls the growth of hair, and its most prominent feature is regeneration. The initiation of morphogenesis of primary and secondary hair follicles in cashmere goats occurs at different stages of embryonic development, and the initiation of primary hair follicles is earlier than that of secondary hair follicles. At the embryonic stage of 4555 days, the skin forms a complete epidermal structure, and the hair follicles have not yet appeared; at 55-65 days, the primary hair follicles begin to develop in various parts of the fetus, and the keratinocytes in the basal layer of the epithelium are arranged in a fence to form hair buds, but the formation of primary hair follicles in the lateral part of the body is later than that in other parts (such as the top of the head, shoulder, and neck). At 65 days, obvious primary follicle hair buds are observed on the sides of the body. At 65-75 days, the primordial bodies of secondary hair follicles are observed in various parts of the fetus, and secondary hair follicles begin to occur and grow from the epidermis near the primary hair follicles. Similar to the primary hair follicles, the formation of secondary hair follicles in the lateral part of the body is later than that in other parts. At 75 days, obvious secondary hair follicle hair buds are observed on the side of the body (Supplementary Figure 1; Zhang et al., 2006, 2007).

The hair follicle traits of cashmere goats have a direct and important effect on the quantity and quality of cashmere. The ultimate goal of the research in the field of hair follicle growth and development in cashmere goats is to reveal the mechanism of cashmere growth and find the important genes related to cashmere growth. The morphogenesis and development of hair follicles may be related to some proteincoding genes. At present, it is considered that most of the signaling molecules regulating hair follicle morphogenesis belong to the Wnt pathway (Li et al., 2004), tumor necrosis factor (TNF) family, fibroblast growth factor (FGF) family (Milla, 2002), bone morphogenetic protein (BMP) family (Thomadakis et al., 1999), Sonic hedgehog (SHH) conduction pathway (McMahon et al., 2003), transforming growth factor

Abbreviations: CircRNA, circular RNA; ceRNA, competing endogenous RNA; qRT-PCR, quantitative RT-PCR.
(TGF) family (Ullrich and Paus, 2005), and NOTCH conduction pathway (Crowe et al., 1998). Some of these coding genes are stimulants of hair follicle development and some are inhibitors, which are repeatedly used to regulate each other.

miRNA is an early non-coding RNA in hair follicles. Researchers identified 22 new miRNAs and 316 conserved miRNAs in adult Inner Mongolia cashmere goats and speculated that miRNA-203 may play an important role in the growth of skin and hair follicles (Liu et al., 2012), and verified that miRNA-203 may regulate the development of cashmere goat hair follicles by targeting DDOST and NAE1 (Ma et al., 2021). In recent years, the role of IncRNA in skin and hair follicles has been gradually a concern of researchers. Studies have found that lncRNA-000133 has a complex regulatory relationship with related miRNAs and their target genes. Overexpression of lncRNA-000133 leads to a significant increase in the relative expression of ET-1, SCF, ALP, and LEF1 in dermal papilla cells (Zheng et al., 2019); lncRNA599547 regulates the expression of Wnt10b gene by targeting miR-15b-5p, thus, inducing the differentiation of dermal papilla cells (Yin et al., 2020).

Circular RNA (circRNA) is a special kind of RNA, which has no free $5^{\prime}$ cap structure and $3^{\prime}$ poly (A) structure, and is insensitive to nuclease (William and Norman, 2014). CircRNAs can be divided into four types according to its origin: intron circRNA, exon circRNA, exon-intron circRNA, and intergenic circRNA (Chen and Li, 2015). The main mechanisms of action of circRNAs include regulating the expression of the host genes (Zhang et al., 2013; You et al., 2015), interacting with RNA-binding proteins ( $\mathrm{Xu}$ et al., 2018); translating proteins (Conn et al., 2015); and acting as competitive endogenous RNA to regulate the expression of genes (Hansen et al., 2013; Memczak et al., 2013; Westholm et al., 2014). A current research focus is the action of circRNA, through competitive binding to miRNA, in regulating gene expression to complete the regulation of life activities that has become a research hotspot. Studies have shown that circLMO7 can enhance the HDAC4 expression of the miR-378a-3p target gene through competitive binding of miR-378a-3p, promote muscle cell proliferation, and inhibit the differentiation of bovine myoblasts (Wei, 2017); CircARF3 adsorbs miR-103, to alleviate the targeted inhibition of miR-103 on TRAF3 and alleviate adipose inflammation by promoting mitochondrial autophagy (Zhang, 2018). CircRNA3669 as competing endogenous RNA (ceRNA) adsorbs miR-26a and removes the downregulation of RCN2 by miR-26a in dairy goat endometrial epithelial cells (Liu, 2019). However, circRNA studies on the regulation of hair follicle development in the embryonic stage of cashmere goats are still scarce. 
In the previous research of our group, we analyzed the regulatory role of miRNA-mRNA in the development of hair follicles at embryo stage in cashmere goats (Han et al., 2020). In this study, in order to explore the pattern of expression and functional role of circRNAs in the development of fetal hair follicles of cashmere goats, we first used a high-throughput sequencing technique to construct the circRNA expression profiling of cashmere goats during the fetal period $(45,55,65$, and 75 days), and identified the DE circRNAs in different comparison groups. At the same time, the host genes of DE circRNAs were analyzed by Gene Ontology (GO) and the Kyoto Encyclopedia of Genes and Genomes (KEGG) pathway. Following this, the regulatory network of circRNA-miRNA-mRNA was constructed by combining miRNA and mRNA databases, and the binding of circRNA-miRNA was verified by dual-luciferase reporter assays. This study has laid a foundation for further exploration of the regulatory role of circRNAs as ceRNAs in the hair follicles of cashmere goats and has also provided a new direction for the study of hair follicles development.

\section{MATERIALS AND METHODS}

\section{Animals and Samples}

In this experiment, 12 3-year-old ewes with good production performance, the same growth environment, and the same feed were selected for breeding in Inner Mongolia Jinlai Animal Husbandry Technology Co., Ltd. (Hohhot, Inner Mongolia), and the breeding time was recorded. The environment of the cashmere goat farm meets the relevant requirements of the experimental facilities in the Chinese national standard "Experimental Animal Environment and Facilities" (GB149252010). Health status, pathogenic microorganism infections, and zoonotic infections were monitored to ensure animal safety and all animal experiments were performed in accordance with the "Guidelines for Experimental Animals" of the Ministry of Science and Technology (Beijing, China). A total of 12 fetal skin samples were collected during the four periods of 45 , 55,65 , and 75 days of gestation of goats, immediately treated with DPEC water, and placed in liquid nitrogen. The samples were then stored in a refrigerator at $-80^{\circ} \mathrm{C}$ for RNA-seq and quantitative RT-PCR (qRT-PCR) tests. All fetal skin samples were collected in accordance with the International Guiding Principles for Biomedical Research Involving Animals and approved by the Special Committee on Scientific Research and Academic Ethics of Inner Mongolia Agricultural University, responsible for the approval of biomedical research ethics of Inner Mongolia Agricultural University [Approval No. (2020) 056]. No specific permissions were required for these activities, and no endangered or protected species were involved.

\section{RNA Library Construction and Sequencing}

This study sequenced 12 samples of lateral skin of cashmere goats at 45, 55, 65, and 75 days of fetal period. Total RNA was isolated and purified using Trizol reagent (Invitrogen, Carlsbad, CA, United States), following the manufacturer's procedure. The amount of RNA and purity of each sample were quantified using NanoDrop ND-1000 (NanoDrop, Wilmington, DE, United States). The RNA integrity was assessed using Agilent 2100. Approximately $5 \mu \mathrm{g}$ of total RNA was used to deplete ribosomal RNA according to the instructions of the Ribo-Zero ${ }^{\text {TM }}$ rRNA Removal Kit (Illumina, San Diego, CA, United States), and the remaining RNA fragments were reverse transcribed using an RNA-seq Library Preparation Kit (Illumina) to form the final cDNA. Finally, we performed the paired-end sequencing on an Illumina Hiseq 4000 (LC Bio, Hangzhou, Zhejiang, China), following the vendor's recommended protocol.

\section{Identification of Transcripts}

According to the characteristics of circRNA structure and splicing sequence, and combined with literature reports, we used CIRCExploter2 (Zhang et al., 2014; Wang et al., 2020) and CIRI (Gao et al., 2015, 2018) to predict circRNAs, and integrate the results of the two software programs according to the starting and ending positions of circRNA. The following is the circRNA identification standard: (1) Mismatch $\leq 2$; (2) back-spliced junctions reads $\geq 1$; (3) two splice sites comprise less than $100 \mathrm{~kb}$ of the genome. According to the above identification and screening criteria, circRNA was identified more accurately.

\section{Differential Expression Analysis}

We used SRPBM as a normalization method to quantify the expression of circRNA.

$\mathrm{SRPBM}=\frac{\mathrm{SR} \times 10^{9}}{N}$, where $\mathrm{SR}$ is the number of spliced reads, and $N$ is the total number of mapped reads in the sample. Analysis of differentially expressed circRNA was done using $\mathrm{R}$ package-edge, differential multiples (fold change) and $P$-values were used by default to screen differential circRNAs; that is, genes that simultaneously satisfy the absolute value of $\log 2$ (fold change) greater than or equal to 1 and $P$-value less than or equal to 0.05 are marked as yes; otherwise, they are marked as no.

\section{Gene Ontology and Kyoto Encyclopedia of Genes and Genomes Pathway Enrichment Analysis}

Gene Ontology and KEGG enrichment analysis is the use of all GO and KEGG annotated circRNA host genes. The gene ontology database $^{1}$ was used to perform functional annotations on DE transcripts of three components, namely, biological processes (BPs), molecular function (MF), and cellular component (CC). Pathway significant enrichment analysis can identify that the host genes are involved in the major biochemical metabolic pathways and signal transduction pathways using the KEGG database ${ }^{2}$. The hypergeometric test was used to analyze GO enrichment of host genes and the statistical enrichment of host genes in KEGG pathways. GO terms and KEGG pathways (corrected $P$-value $<0.05)$ were considered significantly enriched.

\footnotetext{
${ }^{1}$ http://www.geneontology.org/

${ }^{2}$ http://www.genome.jp/kegg/
} 


\section{Construction of CircRNA Regulatory Networks}

In this study, two software programs, Targetscan ${ }^{3}$ and miRanda ${ }^{4}$, were used to predict the miRNA targeted by circRNA. The target genes targeting miRNA were predicted, and the circRNA-miRNA-mRNA regulatory network was constructed preliminarily. Finally, Cytoscape (Shannon et al., 2003) was used to visualize it.

\section{qRT-PCR}

In accordance with the manufacturer's instructions, we used Trizol reagent (Takara, Dalian, Liaoning, China) to extract the total RNA from 12 skin samples representing the fetal periods of cashmere goats. Subsequently, we used a PrimeScript RT Reagent Kit with gDNA Eraser (Takara, Dalian, Liaoning, China) to reverse transcribe RNA to cDNA. Following this, each sample with three duplicates, was tested on a LightCycler ${ }^{\circledR} 96$ Real-Time PCR system (Roche, Basel, Switzerland) using TB GreenPremix Ex Taq II (Takara, Dalian, Liaoning, China). The qRT-PCR conditions were: $95^{\circ} \mathrm{C}$ for $30 \mathrm{~s}$ and then 40 cycles at $95^{\circ} \mathrm{C}$ for $10 \mathrm{~s}, 60^{\circ} \mathrm{C}$ for $30 \mathrm{~s}$, followed by $72^{\circ} \mathrm{C}$ for $10 \mathrm{~s}$. $\beta$-actin was used as the reference gene (Shen et al., 2020; Zhang et al., 2020). The validated primers used for RT-qPCR are listed in the Supplementary Table 1 . The expression levels were calculated using the $2^{-\Delta \Delta C T}$ method (Schmittgen and Livak, 2008).

\section{Dual-Luciferase Reporter Assays}

Chi-miR-27b-3p mimics and chi-miR-16a-3p mimics were synthesized by Hanbio Biotechnology Company (Shanghai, China). The miRNA mimics were transfected into HEK 293T cells using the LipoFiter transfection reagent according to the manufacturer's instructions. The psiCHECK2-circRNA3236-WT construct was generated by inserting the circRNA3236 fragments containing the miRNA binding sequence into the psiCHECK2 vector (Promega) at the $3^{\prime}$ end of the Renilla luciferase gene. The mutant psiCHECK2-circRNA3236-MUT construct was generated by mutating the miRNA-binding sequence to the complementary sequence using overlapping extension PCR. For circRNA3236 luciferase assays, the HEK 293T cells were transfected with miRNA mimics and either the psiCHECK2circRNA3236-WT or mutated psiCHECK2-circRNA3236-Mut reporter plasmid. At $48 \mathrm{~h}$ post-transfection, luciferase activity was measured using a dual-luciferase reporter assay system (Promega) according to the manufacturer's instructions. The relative luciferase activities were calculated by comparing the Firefly/Renilla luciferase activity ratio.

\section{Statistical Analysis}

SPSS18.0 (Beijing, China) was used to calculate the Spearman correlation coefficient and dual-luciferase assay. Results are expressed as the mean \pm SEM, and statistically significant differences between two means were analyzed using $t$-test. A value of $P<0.05$ was considered statistically significant.

${ }^{3}$ http://www.targetscan.org/mamm_31/

${ }^{4}$ http://www.microrna.org/microrna/home.do

\section{RESULTS}

\section{Identification and Characterization of CircRNAs in Hair Follicles of Cashmere Goats}

In order to explore the expression pattern of circRNA in fetal skin hair follicles of cashmere goats, in this study, highthroughput sequencing was performed at four stages of the fetal phase of Inner Mongolian cashmere goats (Albas type). First, we constructed 12 libraries that removed ribosomal RNA of cashmere goats during the fetal period, which were named d45_1, d45_2, d45_3, d55_1, d55_2, d55_3, d65_1, d65_2, d65_3, d75_1, d75_2, and d75_3, respectively. These libraries were applied to the IlluminaHiseq4000 platform for RNA sequencing, and 1,063,299,566 raw reads were obtained from the 12 libraries (Table $\mathbf{1}$ ).

In these original reads, $1,023,889,360$ effective reads were obtained by removing the reads with a connector (adaptor); the reads with $\mathrm{N}$ ( $\mathrm{N}$ indicating that the base information cannot be determined) are greater than $5 \%$, and those are of low quality (the bases with a quality value $\mathrm{Q} \leq 10$ accounting for more than $20 \%$ of the total reads) (Table 2). The Q20 (the proportion of bases with quality value $\geq 20$, error rate $<0.001$ ) of each library was $99.90 \%$ and the Q30 (the proportion of bases with quality value $\geq 30$, error rate $<0.001$ ) was above $98.1 \%$ (Table 1 ), indicating that the sequencing accuracy was high. Comparing the $1,023,889,360$ effective reads with the reference genome, the percentage of the number of reads on the reference genome as a percentage of valid reads was more than $94 \%$, the percentage of the number of reads compared with the unique location of the reference genome as a percentage of valid reads was more than $77 \%$, and the number of reads compared with the multiple locations of the reference genome as a percentage of valid reads was more than $17 \%$. Therefore, the utilization rate of the data was normal, and the original data obtained met the requirements of the subsequent circRNA analysis (Table 2) in terms of quantity and quality.

According to the characteristics of the circRNA structure and splicing sequence, we used CIRCExploter2 and CIRI to predict circRNAs. The results showed that the total number of specific circRNA identified in each sample was more than 2,800, and the corresponding parental genes numbered more than 1,700 (Figure 1). Studies have found that most circRNAs contain two to four exons (Figure 2A). At the same time, the exon length of circRNAs indicates that the length of a single exon is longer than that of circRNAs composed of multiple exons (Figure 2B). Chromosome distribution analysis showed that circRNAs were distributed on almost all chromosomes, and the number of circRNAs on chromosomes 1, 10, and 11 was higher than those on other chromosomes (Figure 2C). Finally, exon circRNAs accounted for 92.01\% (Figure 2D) of all circRNAs in the cashmere goat skin hair follicles.

\section{Analysis of Differences in CircRNAs}

In order to explore further the regulatory role of circRNAs in the early development of cashmere goat hair follicles, we 
TABLE 1 | Data quality control statistics.

\begin{tabular}{|c|c|c|c|c|c|c|c|c|}
\hline \multirow[t]{2}{*}{ Sample } & \multirow{2}{*}{$\frac{\text { Raw data }}{\text { Read }}$} & \multicolumn{3}{|c|}{ Valid data } & \multirow[t]{2}{*}{ Valid ratio (reads) } & \multirow[t]{2}{*}{ Q20 \% } & \multirow[t]{2}{*}{ Q30 \% } & \multirow[t]{2}{*}{ GC content $\%$} \\
\hline & & Base & Read & Base & & & & \\
\hline d45_1 & $83,377,840$ & $12.51 \mathrm{G}$ & $80,991,260$ & $12.15 \mathrm{G}$ & 97.14 & 99.97 & 98.33 & 46 \\
\hline d45_2 & $80,073,650$ & $12.01 \mathrm{G}$ & $77,655,516$ & $11.65 \mathrm{G}$ & 96.98 & 99.97 & 98.33 & 45.50 \\
\hline d45_3 & $91,499,974$ & $13.72 \mathrm{G}$ & $87,070,648$ & 13.06G & 95.16 & 99.98 & 98.41 & 46 \\
\hline d55_1 & $100,354,248$ & $15.05 \mathrm{G}$ & $96,199,486$ & $14.43 G$ & 95.86 & 99.98 & 98.45 & 46.50 \\
\hline d55_2 & $91,779,488$ & $13.77 \mathrm{G}$ & $88,089,256$ & $13.21 \mathrm{G}$ & 95.98 & 99.98 & 98.53 & 47 \\
\hline d55_3 & $94,888,486$ & $14.23 \mathrm{G}$ & $91,438,886$ & $13.72 \mathrm{G}$ & 96.36 & 99.98 & 98.48 & 47 \\
\hline d65_1 & $90,684,330$ & $13.60 \mathrm{G}$ & $87,483,330$ & $13.12 \mathrm{G}$ & 96.47 & 99.97 & 98.17 & 47 \\
\hline d65_2 & $102,708,880$ & $15.41 \mathrm{G}$ & $99,047,498$ & $14.86 \mathrm{G}$ & 96.44 & 99.98 & 98.47 & 46.50 \\
\hline d65_3 & $80,732,910$ & $12.11 \mathrm{G}$ & $77,823,688$ & $11.67 \mathrm{G}$ & 96.40 & 99.97 & 98.34 & 47 \\
\hline d75_1 & $81,140,600$ & $12.17 \mathrm{G}$ & $77,983,860$ & $11.70 \mathrm{G}$ & 96.11 & 99.98 & 98.45 & 45 \\
\hline d75_2 & $83,141,742$ & $12.47 \mathrm{G}$ & $80,115,068$ & $12.02 \mathrm{G}$ & 96.36 & 99.98 & 98.46 & 45 \\
\hline d75_3 & $82,917,418$ & $12.44 G$ & $79,990,864$ & $12.00 \mathrm{G}$ & 96.47 & 99.97 & 98.33 & 46 \\
\hline
\end{tabular}

TABLE 2 | Reference genome alignment read statistics.

\begin{tabular}{|c|c|c|c|c|}
\hline Sample & Valid reads & Mapped reads (\%) & Unique mapped reads (\%) & Multi mapped reads (\%) \\
\hline d45_1 & $80,991,260$ & 76,343,633 (94.26) & $61,948,389(76.49)$ & $14,395,244(17.77)$ \\
\hline d45_2 & $77,655,516$ & $74,877,365(96.42)$ & $61,031,456(78.59)$ & $13,845,909(17.83)$ \\
\hline d45_3 & $87,070,648$ & $84,828,546(97.42)$ & $68,283,000(78.42)$ & $16,545,546(19.00)$ \\
\hline d55_1 & $96,199,486$ & $93,762,003$ (97.47) & $76,182,223$ (79.19) & $17,579,780(18.27)$ \\
\hline d55_2 & $88,089,256$ & $85,888,657$ (97.50) & $69,725,622(79.15)$ & 16,163,035 (18.35) \\
\hline d55_3 & $91,438,886$ & $89,149,678(97.50)$ & $71,925,770(78.66)$ & $17,223,908(18.84)$ \\
\hline d65_1 & $87,483,330$ & $85,122,572(97.30)$ & $68,170,805$ (77.92) & $16,951,767$ (19.38) \\
\hline d65_2 & $99,047,498$ & $96,402,904$ (97.33) & $79,282,917$ (80.05) & $17,119,987(17.28)$ \\
\hline d65_3 & $77,823,688$ & $75,757,511$ (97.35) & $60,872,547$ (78.22) & $14,884,964$ (19.13) \\
\hline d75_1 & $77,983,860$ & 75,996,848 (97.45) & $62,127,737$ (79.67) & $13,869,111(17.78)$ \\
\hline d75_2 & $80,115,068$ & $78,137,926$ (97.53) & $64,459,926(80.46)$ & 13,678,000 (17.07) \\
\hline d75_3 & $79,990,864$ & $77,918,087$ (97.41) & $63,626,735$ (79.54) & $14,291,352(17.87)$ \\
\hline
\end{tabular}

divided the four stages into six comparison groups and analyzed the differential expression by calculating the SRPBM value of circRNAs (Figures 3A,B). The results are as follows: d75vsd45, circRNA upregulated by 59 and downregulated by 33 ; d75vsd55, circRNA upregulated by 61 and downregulated by 102 ; d75vsd65, circRNA upregulated by 32 and downregulated by 33; d65vsd55, circRNA upregulated by 67 and downregulated by 169 ; d65vsd 45 , circRNA upregulated by 96 and downregulated by 63; and d55vsd45, circRNA upregulated by 76 and downregulated by 42 (Figure 4). By further exploring the law of differential expression of circRNA, it was found that the differential expression of circRNA of d65vsd55 was the greatest, while the differential expression of circRNA of d75vsd65 was the lowest (Figure 4 and Supplementary Figure 2).

\section{Validation of CircRNAs by qRT-PCR}

To validate the accuracy of the circRNA sequencing results, the relative expression of six DE circRNAs (circRNA2049, circRNA3411, circRNA2225, circRNA5681, circRNA1604, and circRNA4351) (Supplementary Table 2), were measured by qRTPCR (Figure 5). The qRT-PCR results were consistent with the transcriptome sequencing data.

\section{Gene Ontology and Kyoto Encyclopedia of Genes and Genomes Pathway Analysis of Host Genes}

Gene Ontology analysis includes three domains describing the cellular and molecular roles of genes and gene products (BP, CC, and MF) (Harris et al., 2004). KEGG is a pathway database for the systematic analysis of gene function, linking genomic and functional information (Ogata et al., 1999). In order to explore the regulatory role of host genes of $\mathrm{DE}$ circRNAs in hair follicle genesis and development in cashmere goats, we analyzed the host genes of circRNA that were differentially expressed in different control groups by GO and KEGG pathway analyses. The results of GO enrichment showed that there was gene enrichment in the BPs related to the growth and development of hair follicles, such as hair follicle morphogenesis (GO:0031069), cell development (GO:0048468) MF including TGF beta receptor binding (GO:0005160), repressing transcription factor binding (GO:0070491), and CC including cell junction (GO:0030054), and transcription elongation factor complex (GO:0008023). A total of 54 pathways were significantly enriched in the six comparison groups. KEGG pathway analysis showed that there was gene enrichment in the 


\section{Identification results of circRNA}

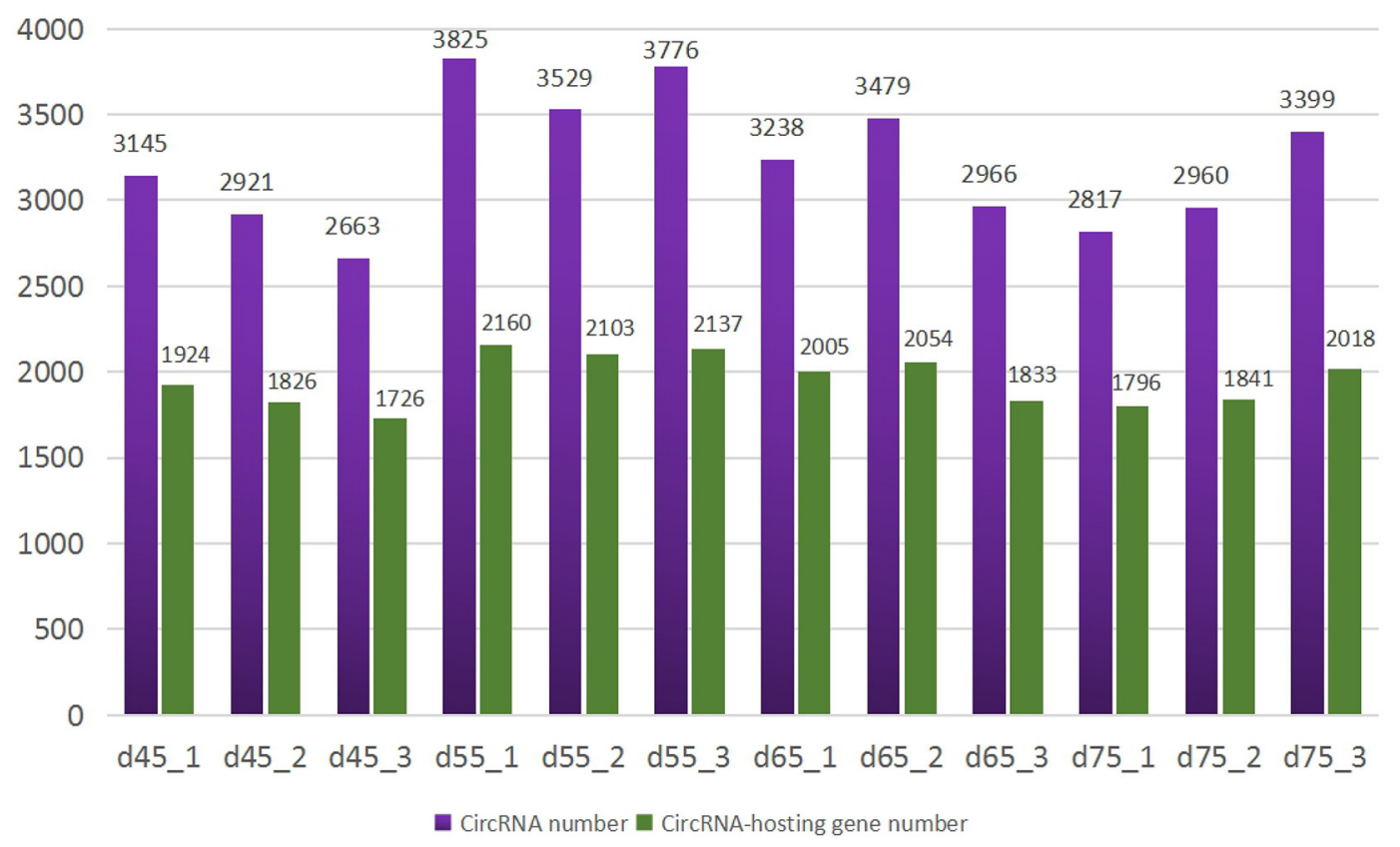

FIGURE 1 | Identification results of circRNAs. The purple columns represent the number of circRNAs, and the green columns represent the number of circRNA-hosting genes.

Notch signaling pathway (ko04330), NF- $\mathrm{B}$ signaling pathway (ko04064), PI3K-AKt signaling pathway (ko04151), and other signal pathways (Supplementary Figure 3). Therefore, the host genes corresponding to differentially expressed circRNAs may be involved in the process of hair follicle growth and development, and then play a regulatory role.

\section{Functional Analysis of CircRNA as an miRNA Sponge}

The ceRNA hypothesis is a new model for post transcriptional gene regulation. According to the hypothesis, the expression of designated miRNAs is reduced by ceRNA (Wang et al., 2019). To construct the ceRNA network of circRNA-miRNA-mRNA, we integrated our miRNA library data (unpublished data) and mRNA library data to analyze the miRNA binding sites in the circRNAs and mRNA using miRanda and Targetscan. We selected DE exon circRNA in d75vsd45 for construction of the ceRNA regulatory network. In the up-down-up regulation pattern, we predicted 46 circRNA-miRNA and 49 miRNAmRNA interactions (Supplementary Table 3 and Figure 6A). As shown in Figure 6A, upregulated circRNA9106 may serve as a sponge for multiple miRNAs (chi-miR-1, chi-miR-18a-3p, and chi-miR-93-3p). Notably, three circRNAs, circRNA8058, circRNA6363, and circRNA8624 contained seed targets of chi-miR-133a-5p, which were identified by searching for miRNA target sites. Moreover, these miRNAs can downregulate the expression of their target genes (chi-miR-133a-5p was predicted to bind with FLRT1, SOX5, and RBPJL genes). We further constructed a down-up-down co-expression network using miRanda and Targetscan with a strict model, for which 56 circRNA-miRNA and 77 miRNA-mRNA interactions were predicted (Supplementary Table 4 and Figure 6B). In the down-up-down co-expression network, three downregulated circRNAs, circRNA3382, circRNA1448, and circRNA1896, contained seed targets of chi-miR-26b-3p. chi-miR-26b-3p was predicted to bind with multiple target genes, including MCTP1, MEF2C, GPC6, and FZD5. At the same time, circRNA3236 was predicted to have two target miRNAs (chi-miR-27b-3p and chi-miR-16b-3p).

\section{CircRNA3236 Binds to chi-miR-27b-3p and chi-miR-16b-3p}

CircRNA3236 was downregulated in d75vsd45; Targetscan and miRanda software predicted that circRNA3236 was targeted to chi-miR-27b-3p and chi-miR-16b-3p, and there were two binding sites, respectively. Therefore, two mutant vectors were constructed to verify the specific binding sites (Figures 7A,B). The results showed that compared with the NC group, chimiR-27b-3p and chi-miR-16b-3p significantly decreased the expression of luciferase in circRNA3236 WT $(P<0.001)$. It shows that there is a binding effect between the two in this experiment. After mul mutation, chi-miR-27b-3p failed to downregulate the expression of luciferase in circRNA3236mut1 $(P>0.05)$, indicating that the mutation was successful. Mul is the binding site of chi-miR-27b-3p and circRNA3236wt. After mu4 mutation, chi-miR-16b-3p failed to downregulate 

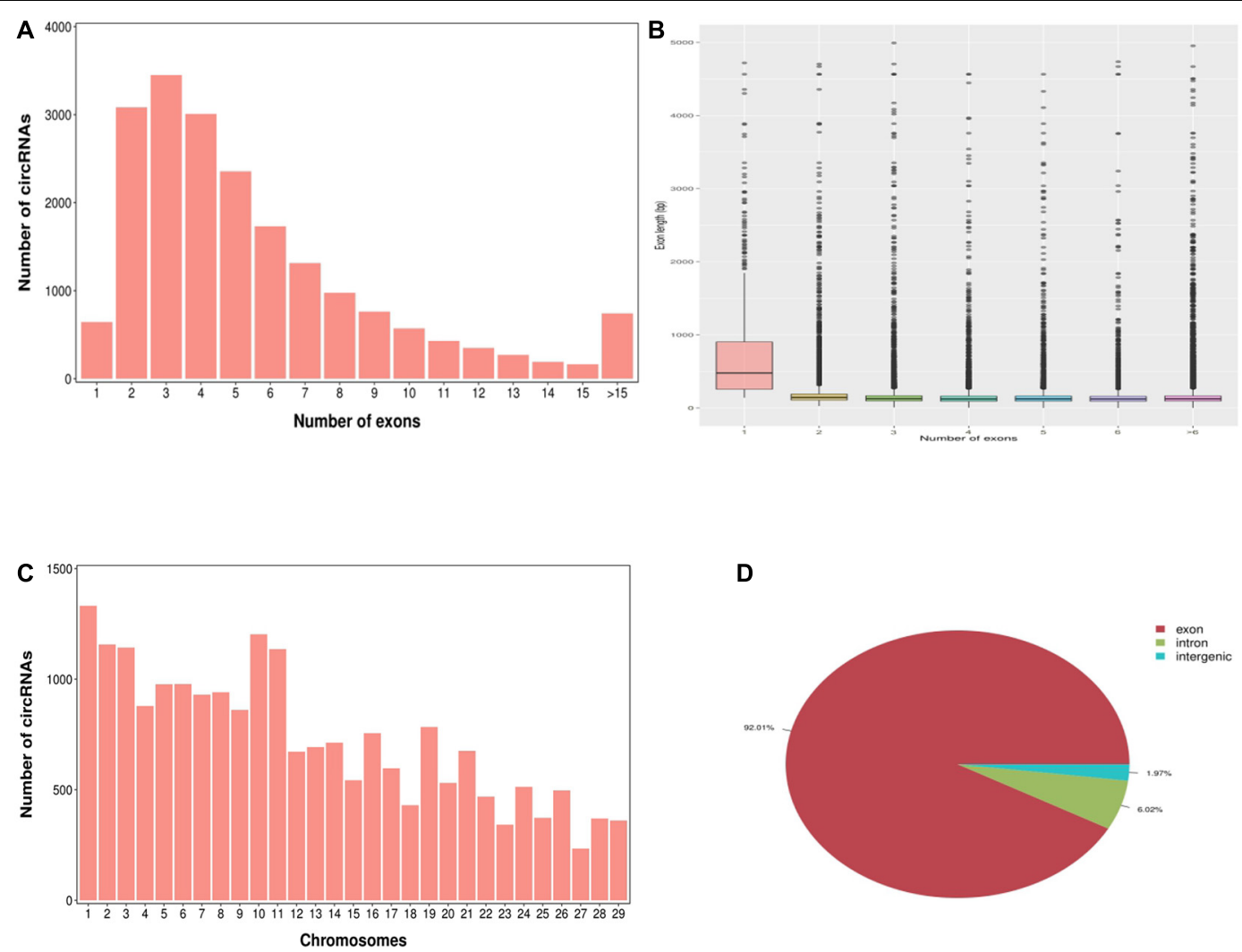

FIGURE 2 | Characteristics of circRNAs in hair follicles of cashmere goats. (A) Distribution of the number of circRNAs per gene. The $x$-axis represents the number of circRNAs/host gene and the $y$-axis represents the number of circRNA. (B) Box plot showing the exon length of exon-derived circRNAs. The $x$-axis represents the number of exons that the circRNA contains and the $y$-axis represents the exon length. (C) Distribution of the identified circRNAs in each chromosome. The $x$-axis represents the number of chromosomes and the $y$-axis represents the number of circRNAs classified by different chromosomes. (D) Classification of circRNAs in hair follicles of cashmere goats.

the expression of luciferase in circRNA236-mut4 $(P>0.05)$, indicating that the mutation was successful. Mu4 is the binding site of chi-miR-16b-3p and circRNA3236 (Figures 7C,D).

\section{DISCUSSION}

Studies have shown that circRNAs may affect biological function by regulating the level of linear mRNA expression (Kelly et al., 2015). In this experiment, the host genes of differential circRNAs were analyzed by GO and the KEGG pathway. The BP of GO enrichment includes hair follicle morphogenesis, hair follicle maturation, and cell growth; the pathway of KEGG enrichment includes the Notch signaling pathway and NF-kappa B signaling pathway. Previous studies have shown that there is a direct relationship between the Notch signal pathway and hair follicle morphogenesis (Lin et al., 2000), high expression of Notch1 and Notch 2 can accelerate the formation of mouse hair substrate, and at the same time, it can inhibit the cells around the hair substrate to form substrate (Crowe et al., 1998). Ocu-miR-205 can promote the transition of Rex rabbit hair follicles from the growing stage to a degenerative quiescent stage by regulating the expression of related genes and proteins in Notch, BMP, and other signaling pathways, to change the hair density (Liu et al., 2020). Krieger used a mouse model to study the regulation of the NF-kappa B signaling pathway in the hair follicle cycle and found that the NF-kappa B signaling pathway is essential for the growth and activation of hair follicle stem cells (Krieger et al., 2018). The research of the NF-kappa B signaling pathway in mice showed that the NF-kappa B signaling pathway was activated downstream of EdaA1 and EDAR, thus, playing an important role in the development of hair follicles (Schmidt-Ullrich et al., 2006). Zhang et al. (2009) found that $\mathrm{Wnt} / \beta$-catenin signaling transduction is a necessary signaling for NF-kappa B activation, and EDAR is the direct target gene of $\mathrm{Wnt} / \beta$-catenin signaling pathway. The initial activation of the $\mathrm{Wnt} / \beta$-catenin signaling pathway depends on the activity of EDA/EDAR/NF-kappa B in the prohair substrate of primary hair follicles. The complex interaction and interdependence of $\mathrm{Wnt} / \beta$-catenin and EDA/EDAR/NFkappa $B$ signaling pathways in the initiation and maintenance of primary hair follicle substrate were revealed (Zhang et al., 2009). Therefore, we speculate that circRNA may play a regulatory role in the primary stage of hair follicle development via the Notch signaling pathway and the NF-kappa B signaling pathway.

In the past few decades, there have been many studies on the regulatory role of miRNA and IncRNA in hair follicles (Sun et al., 2010; Liu et al., 2012; Yuan et al., 2013; Zhou, 2016, Wang et al., 2017; Zhou et al., 2018; Zhou, 2018). However, there 

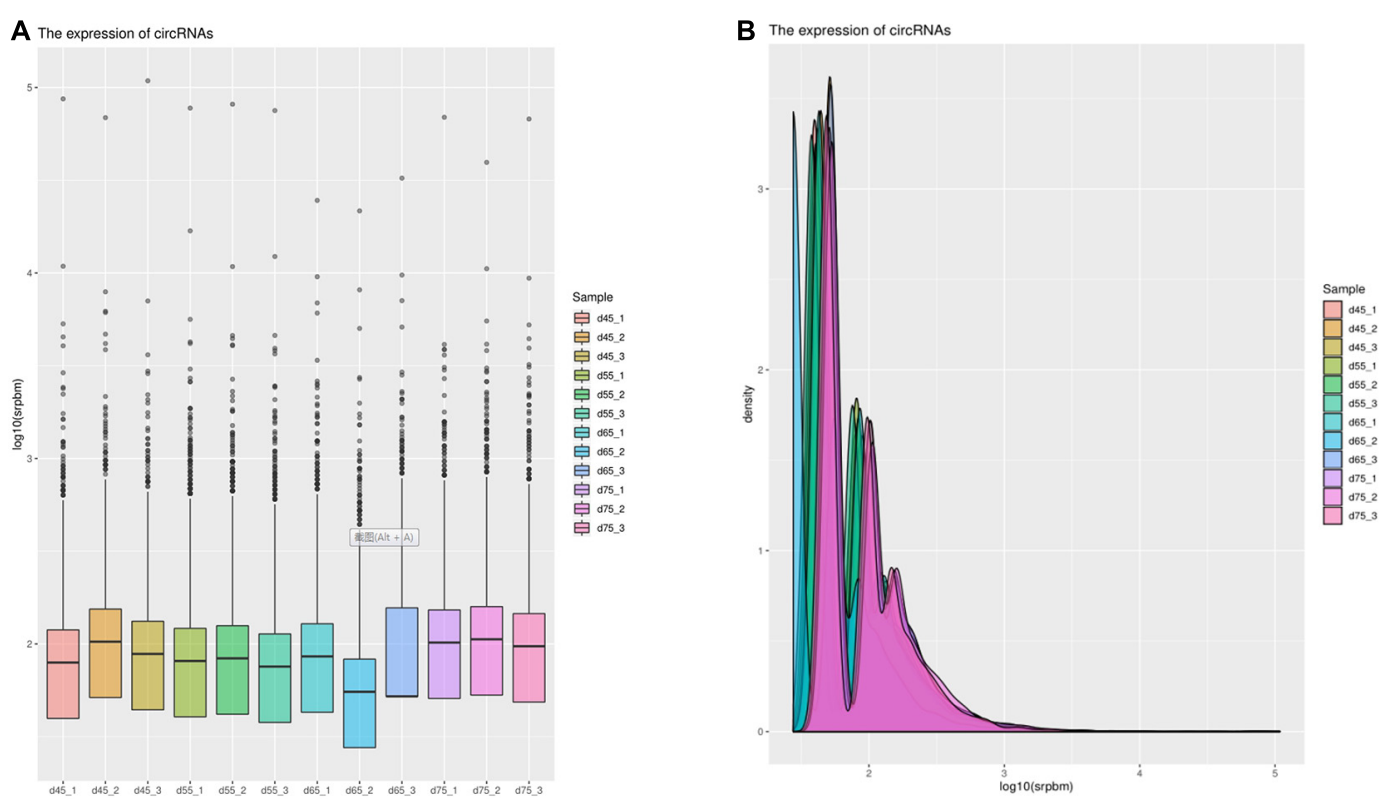

FIGURE 3 | The expression of circRNAs. (A) Box plot showing the expression abundance of circRNAs in each sample. (B) Density plot of the expression density distribution of circRNAs in each sample.

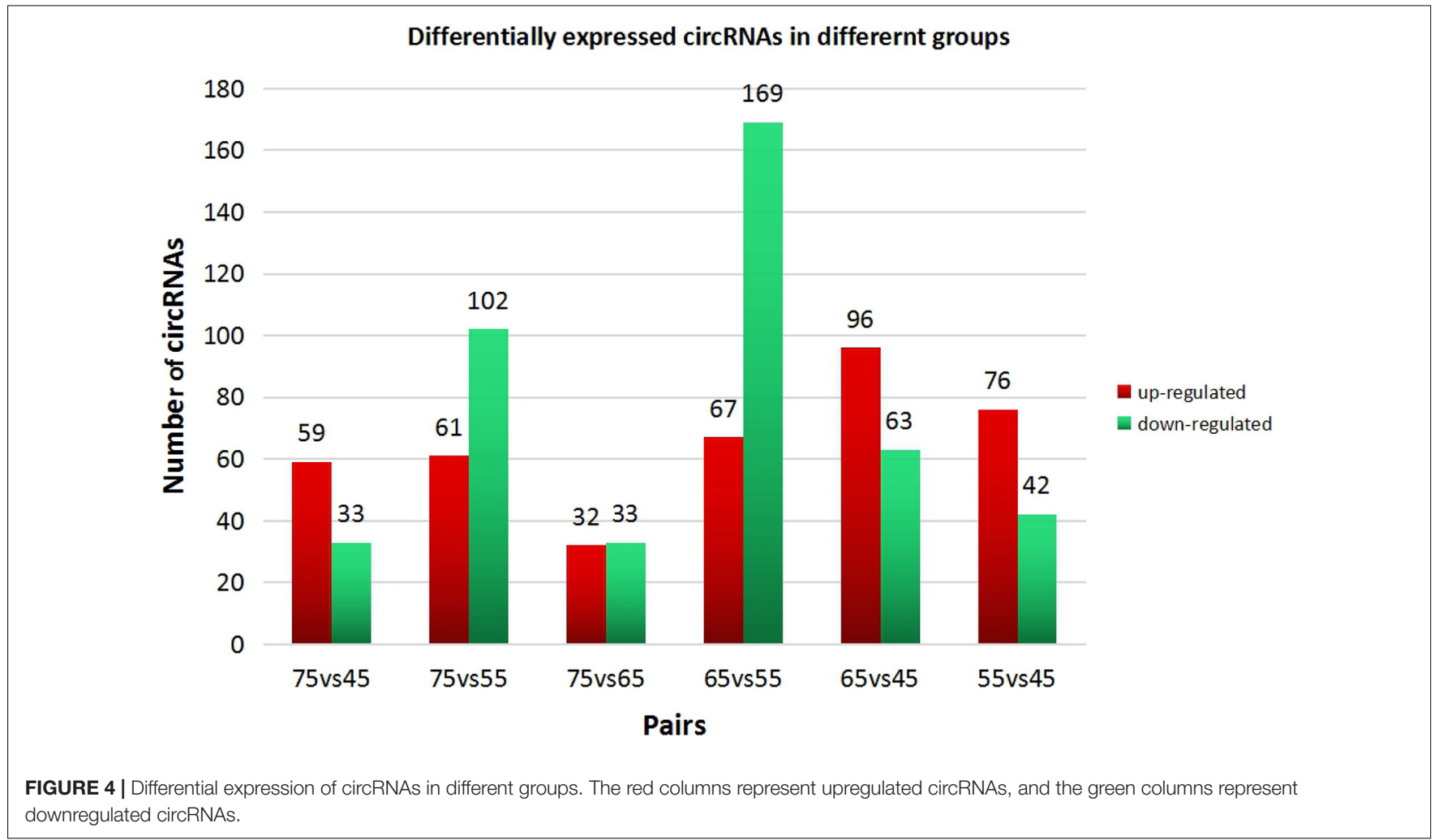

is no report about circRNA related to hair follicle development. CircRNA is mostly located in the cytoplasm, and some circRNAs have MRE (a sequence recognized by miRNA), which can interact with miRNA and participate in molecular regulation as ceRNA. CeRNA was first proposed by the Pandolfi team at Harvard
Medical School in Cell in 2011. It is pointed out that there are competitive endogenous RNA (ceRNA) molecules in cells. These ceRNA molecules (including lncRNA, circRNA, mRNA, pseudogenes, etc.) can compete through miRNA response elements (MRE) for a combination with the same miRNA in 

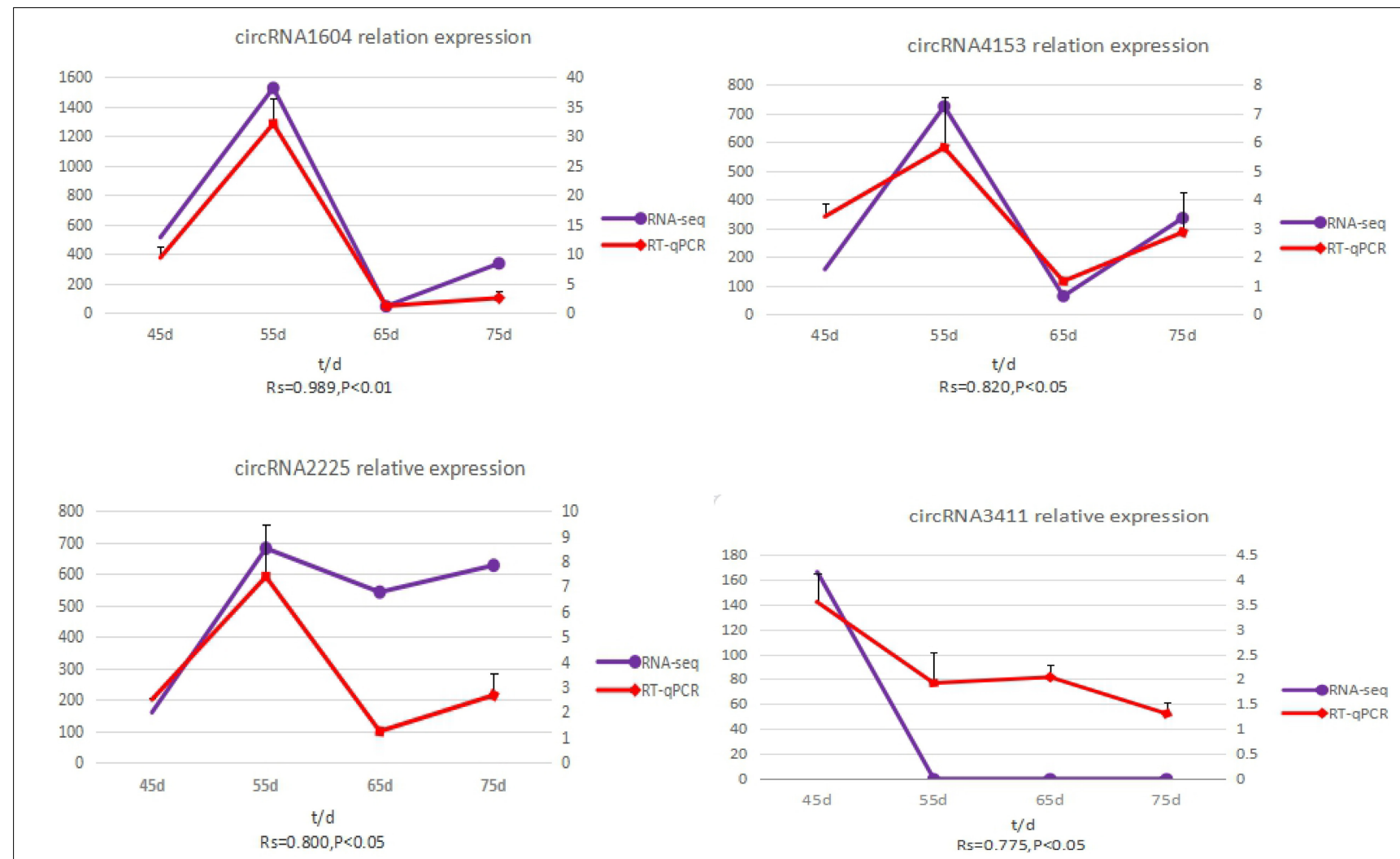

circRNA2049 relative expression
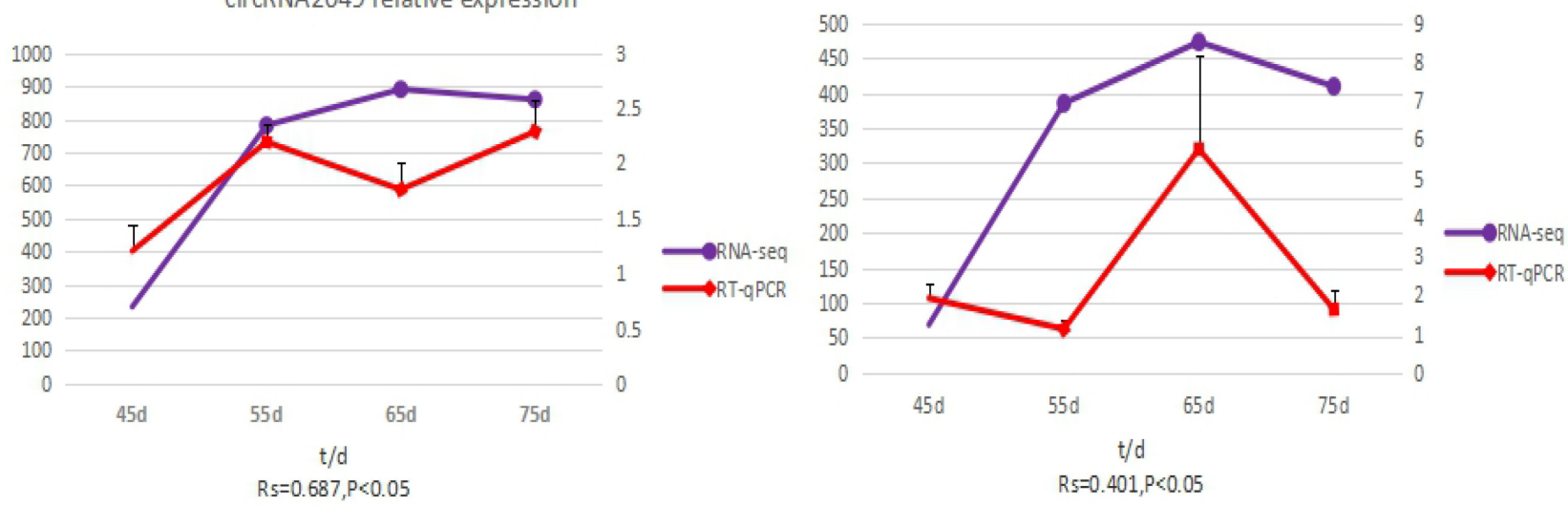

FIGURE 5 | The expression quantity and expression trend of circRNA in different periods. A total of $0.8<\mid$ Rs $\mid<1$ indicates a strong correlation; $0.6<\mid$ Rs $\mid<0.8$ indicates a strong correlation; $0.4<|\mathrm{Rs}|<0.6$ indicates a moderate correlation; $0.2<|\mathrm{Rs}|<0.4$ indicates a weak correlation; $0<|\mathrm{Rs}|<0.2$ indicates no correlation; and the degree of proximity between | Rs| and 1 represents the degree of closeness and correlation between two variables.

order to regulate each other's expression levels (Salmena et al., 2011). In recent years, the involvement of circRNA as ceRNA in the regulation of biological life activities has become the focus of circRNA research. The research mainly focuses on human tumors and the regulation of life activities of some animals. In the field of human tumor research, researchers have constructed the circRNA-miRNA-mRNA regulatory network of liver cancer on the basis of high-throughput sequencing, which provided new insights that circRNA mediates the occurrence and development of liver cancer through a ceRNA mechanism (Zhao J. et al., 2019). It was found that circ_PUM1 could compete with miRNA-136, resulting in upregulation of NOTCH3 expression, thus, promoting the occurrence and development of endometrial carcinoma (Zong et al., 2020). It was identified that hsa_circ_0000467 plays a regulatory role in gastric cancer by regulating the level of miRNA-326-3p, and that circRNA may be a potential diagnostic marker and therapeutic target in gastric cancer (He et al., 2020). It is verified that circFUT8 

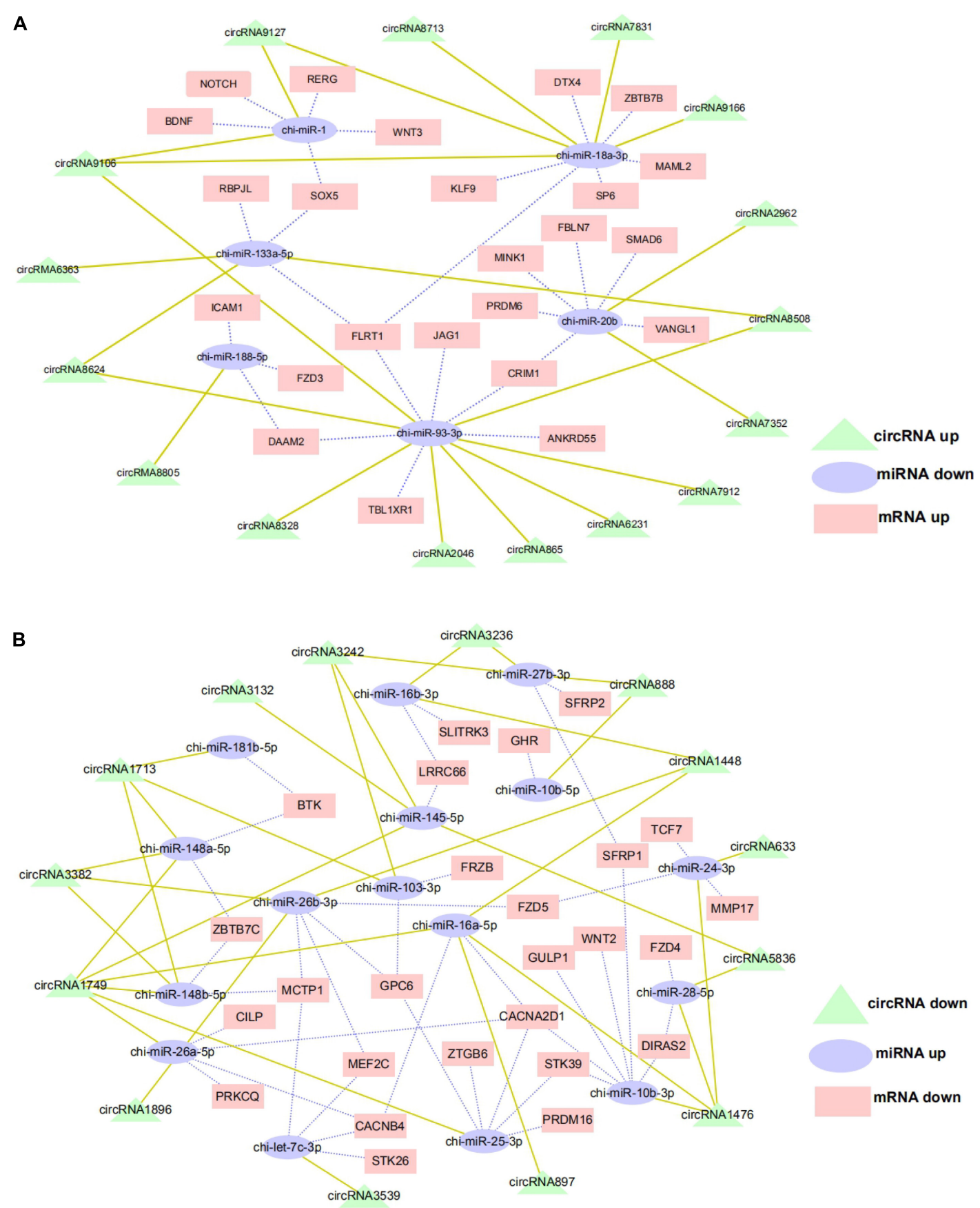

FIGURE 6 | CircRNA-miRNA-mRNA regulatory network analysis in cashmere goat hair follicle. (A) Upregulated circRNA networks and (B) downregulated circRNAs networks for d75vsd45.

plays an inhibitory role in bladder cancer cells by targeting miRNA-570-3p/KLF10 (Mo et al., 2020). In non-human animal research, Zhang et al. (2020) found that circRNA-006258 regulates the growth and lactation of goat mammary epithelial cells through a circRNA-006258-miR-574-5p-EVI5L regulatory network. Wang et al. (2020) constructed a circRNA expression profile related to milk fat metabolism under heat stress and established the related ceRNA regulatory network. Li et al.
(2020) analyzed the differentially expressed circRNA, between fast contractile muscle and slow contractile muscle of porcine skeletal muscle and established a ceRNA network by combining the differentially expressed circRNA with miRNA and mRNA databases, which was preliminarily verified by double luciferase. However, there are no reports on circRNA related to fetal hair follicle development in cashmere goats. In this study, a total of 21,784 circRNA were identified in four stages of fetal skin 

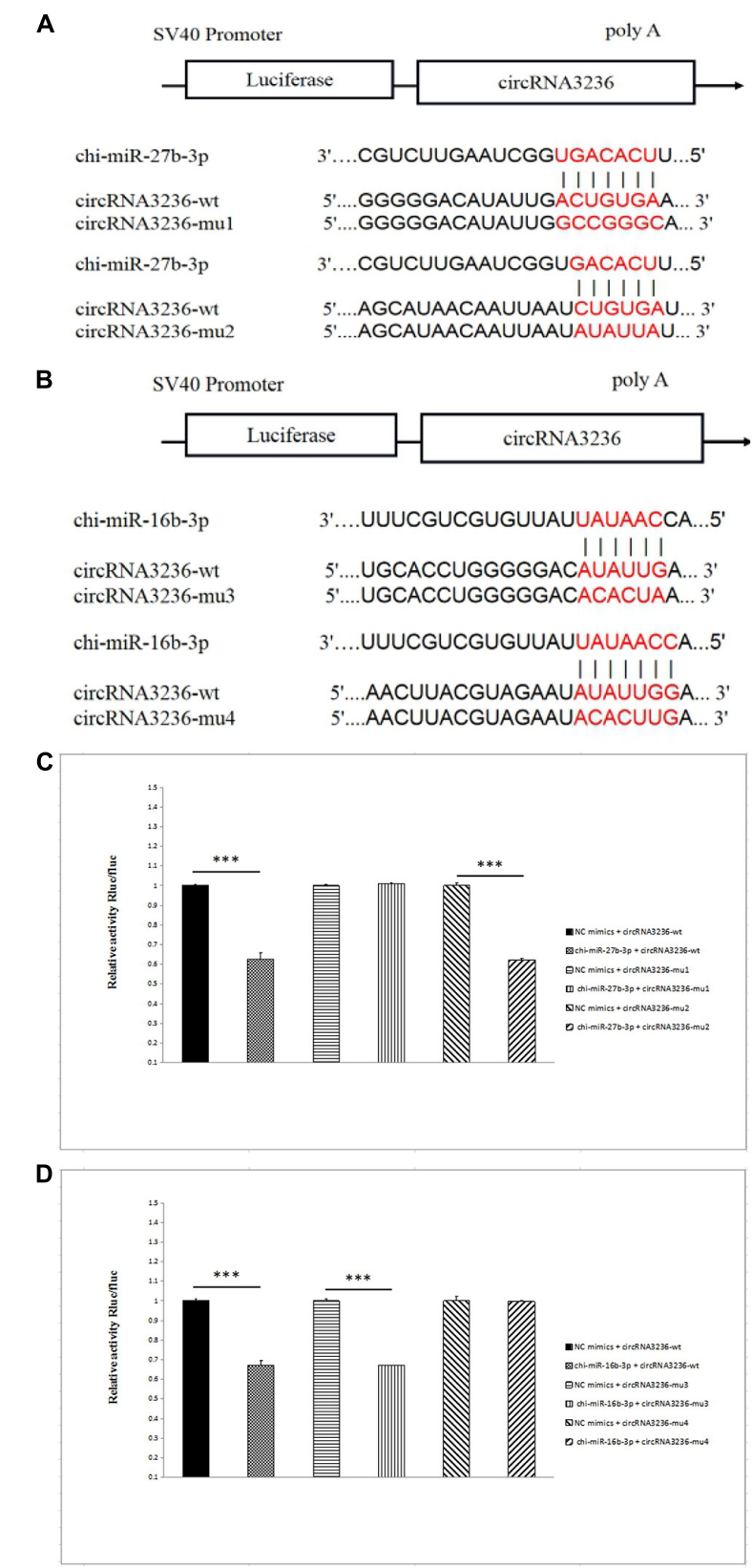

FIGURE 7 | CircRNA3236 sponged with chi-miR-27b-3p andchi-miR-26a-3p. (A) The predicted binding site and mutated site of chi-miR-27b-3p in circRNA3236. (B) The predicted binding site and mutated site of chi-miR-16a-3p in circRNA3236. (C) Detection of interaction between circRNA3236 and chi-miR-27b-3p by dual luciferase reporter gene assay. (D) Detection of interaction between circRNA3236 and chi-miR-16b-3p by dual luciferase reporter gene assay. ${ }^{* * *} P<0.001$ shows that the difference is extremely significant.

hair follicles of cashmere goats. The differentially expressed circRNA of each comparison group was screened, and the differential circRNAs were combined with miRNA and mRNA using the mode of upregulation-downregulation-upregulation or downregulation-upregulation-downregulation to construct the ceRNA network. Some circRNAs and differentially expressed circRNAs at different stages have been identified previously in the hair follicle development of Angora rabbits, but the total number of circRNAs, and differentially expressed circRNAs were greater, and a ceRNA network was established in this study. It is suggested that circRNAs may play an irreplaceable role in the development of hair follicles in cashmere goats (Zhao B.H. et al., 2019).

In order to explore further the functional mechanism of circRNA in the hair follicle development of cashmere goats, we predicted the miRNA targeted by circRNA and the target genes targeted by miRNA, which were differentially expressed by d75vsd45, and, thus, constructed the ceRNA network. Among them, 16 circRNAs targeted six miRNAs, and six miRNAs targeted 23 target genes in the upregulationdownregulation-upregulation model; 14 circRNAs targeted 16 miRNAs, and 16 miRNAs targeted 26 target genes in the downregulation-upregulation-downregulation model. We constructed circRNA2046-chi-miR-93-3p-FLRT1, circRNA2962chi-miR-20b-SMAD6, circRNA3236-chi-miR-27b-3p-SFRP, circRNA3236-chi-miR-16b-3p-SLITRK3, circRNA1476-chimiR-10b-3p-WNT2, and other signaling pathways. Among them, SFRP1 (Hawkshaw et al., 2018), WNT3 (Millar et al., 1999), SMAD6 (Lv et al., 2019), and other genes are all involved in important pathways that have been related to hair follicle development in previous studies, including WNT, TGF- $\beta$, TNF, and other signal pathways. Therefore, we further speculate that circRNA, as ceRNA, may play a regulatory role in fetal skin hair follicles of cashmere goats through related genes such as WNT, TGF- $\beta$, and TNF. The results of the double luciferase experiment show that circRNA3236 and chi-miR27b-3p, and circRNA3236 and chi-miR-16b-3p have a targeted binding relationship. It is suggested that the exon circRNA regulates gene expression by binding miRNA, and then regulates the growth and development of cashmere goat hair follicle. This is consistent with previous studies on the functional mechanism of circRNA.

\section{CONCLUSION}

We constructed the expression profiling of circRNAs in the hair follicles of Inner Mongolia cashmere goats at different embryonic stages $(45,55,65$, and 75 days), and a total of 21,784 circRNA were identified. The results of GO and KEGG analysis of host genes showed that there may be some relationship between circRNA and its host genes, and circRNA may play a role in the $\mathrm{BP}$ of hair follicle growth and development in cashmere goats through the Notch signaling pathway and NF-kappa B signaling pathway. At the same time, the regulatory network of circRNAmiRNA-mRNA was constructed, and the interaction between 102 pairs of circRNA-miRNA and 126 pairs of miRNA-mRNA was studied. The results of the dual luciferase assay showed that circRNA3236 and chi-miR-27b-3p, and circRNA3236 and chimiR-16b-3p have a targeted binding relationship. The specific binding sites were verified, which provides an important basis for exploring the molecular mechanism of circRNA as ceRNA in the morphogenesis and development of hair follicles, and also 
provides important information for studying the mechanism of action of circRNA in human hair follicles.

\section{DATA AVAILABILITY STATEMENT}

The RNA-Seq data were submitted to the SRA database under accession number (SRR13306949, SRR13306948, SRR13306947, SRR13306946, SRR13306945, SRR13306944, SRR13306943, SRR13306942, SRR13306941, SRR13306940, SRR13306939, SRR13306938). Additional data can be found in Supplementary Material.

\section{ETHICS STATEMENT}

All fetal skin samples were collected in accordance with the International Guiding Principles for Biomedical Research Involving Animals and approved by the Special Committee on Scientific Research and Academic Ethics of Inner Mongolia Agricultural University, responsible for the approval of biomedical research ethics of Inner Mongolia Agricultural University (Approval No. [2020] 056). No specific permissions were required for these activities, and no endangered or protected species were involved.

\section{AUTHOR CONTRIBUTIONS}

FS, RM, YJZ, and JL conceived the idea and designed the study. FS, YW, ZXW, LL, and JP participated in the sample collection. FS, RM, EH, ZW, YR, and ZL performed the experiments. ZYW, YHZ, RW, and YJZ analyzed the data. FS and ZD wrote the draft. FS, ZD, YW, and YJZ finalized the manuscript. All authors read and approved the final manuscript.

\section{REFERENCES}

Chen, L. L., and Li, Y. (2015). Regulation of circRNA biogenesis. RNA Biol. 12, 381-388. doi: 10.1080/15476286.2015.1020271

Conn, S. J., Pillman, K. A., Toubia, J., Conn, V. M., Salmanidis, M., Phillips, C. A., et al. (2015). The RNA binding protein quaking regulates formation of circRNAs. Cell 160, 1125-1134. doi: 10.1016/j.cell.2015.02. 014

Crowe, R., Henrique, D., Horowicz, D., and Niswander, L. (1998). A new role for Notch and Delta in cell fate decisions patterning the feather array. Development 125, 767-775. doi: 10.1242/dev.125.4.767

Gao, Y., Wang, J. F., and Zhao, F. Q. (2015). CIRI: an efficient and unbiased algorithm for de novo circular RNA identification. Genome Biol. $16: 4$.

Gao, Y., Zhang, J. Y., and Zhao, F. Q. (2018). Circular RNA identification based on multiple seed matching. Brief Bioinform. 19, 803-810. doi: 10.1093/bib/bbx014

Han, W. J., Yang, F., Wu, Z. H., Guo, F. Q., Zhang, J. J., Hai, E. H., et al. (2020). Inner mongolian cashmere goat secondary follicle development regulation research based on mRNA-miRNA Co-analysis. Sci. Rep. 10:4519.

Hansen, T. B., Jensen, T. I., Clausen, B. H., Bramsen, J. B., Finsen, B., Damgaard, C. K., et al. (2013). Natural RNA circles function as efficient microRNA sponges. Nature 495, 384-388. doi: 10.1038/nature11993

\section{FUNDING}

This study was supported by the Plan Project of Science and Technology in Inner Mongolia (2019GG243), the reported work was supported by the National Natural Science Foundation of China (31860627). The funding played a role in the design of the study and the collection, analysis, and interpretation of data.

\section{ACKNOWLEDGMENTS}

We thank the Inner Mongolia Jinlai Animal Husbandry for providing experimental samples and LC Biological (Huangzhou, China) for the RNA-seq sequencing in this study. We also thank the International Science Editing (http://www. internationalscienceediting.com) for editing this manuscript.

\section{SUPPLEMENTARY MATERIAL}

The Supplementary Material for this article can be found online at: https://www.frontiersin.org/articles/10.3389/fgene. 2021.678825/full\#supplementary-material

Supplementary Figure 1 | Changeable process in hair follicle morphogenesis of the Inner Mongolian cashmere goat in the fetal period.

Supplementary Figure 2 | Volcano plot of all DE circRNAs in six comparison groups.

Supplementary Figure 3 | GO and KEGG enrichment analysis of host genes of DE circRNAs.

Supplementary Table 1 | Primer information for qRT-PCR.

Supplementary Table 2 | CircRNAs verified by qRT-PCR and related information.

Supplementary Table 3 | CircRNA, miRNA, and mRNA in d45 vs d75 were differentially expressed in the up-down-up ceRNA regulatory network.

Supplementary Table 4 | CircRNA, miRNA, and mRNA in d45 vs d75 were differentially expressed in the down-up-down ceRNA regulatory network.

Harris, M. A., Clark, J., Ireland, A., Lomax, J., Ashburner, M., Foulger, R., et al. (2004). The gene ontology (GO) database and informatics resource. Nucleic Acids Res. 32, D258-D261.

Hawkshaw, N. J., Hardman, J. A., Haslam, I. S., Shahmalak, A., Gilhar, A., Lim, X., et al. (2018). Identifying novel strategies for treating human hair loss disorders: cyclosporine a suppresses the Wnt inhibitor, SFRP1, in the dermal papilla of human scalp hair follicles[J]. PLoS Biol. 16:e2003705.

He, Q. Q., Yan, D., Dong, W., Bi, J. M., Huang, L. F., Yang, M. H., et al. (2020). circRNA circFUT8 upregulates Krüpple-like factor10 to inhibit the metastasis of bladder cancer via sponging miR-570-3p. Mol. Ther. Oncolytics 16, 172-187.

Kelly, S., Greenman, C., Cook, P. R., and Papantonis, A. (2015). Exon skipping is correlated with exon circularization. J. Mol. Biol. 427, 2414-2417. doi: 10.1016/ j.jmb.2015.02.018

Krieger, K., Millar, S. E., Mikuda, N., Krahn, I., Kloepper, J. E., Bertolini, M., et al. (2018). NF-kappa B participates in mouse hair cycle control and plays distinct roles in the various pelage hair follicle. J. Invest. Dermatol. 138, 256-264. doi: 10.1016/j.jid.2017.08.042

Li, B. J., Yin, D., Li, P. H., Zhang, Z. K., Zhang, X. Y., Li, H. Q., et al. (2020). Profiling and functional analysis of circular RNAs in porcine fast and slow muscles. Front. Cell Dev. Biol. 8:322. doi: 10.3389/fcell.2020.00322

Li, G. Q., Ji, Y. C., and Li, Y. (2004). Molecular mechanism of hair follicle morphogenesis. Foreign Med. Dermatol. Venereol. 30, 38-40. 
Lin, M. H., Leimeister, C., Gessler, M., and Kopan, R. (2000). Activation of the Notch pathway in the hair cortex leads to aberrant differentiation of the adjacent hair-shaft layers. Development 127, 2421-2432. doi: 10.1242/dev.127.11.2421

Liu, G. Y., Li, S., Liu, H. L., Zhu, Y. L., Bai, L. Y., Sun, H. T., et al. (2020). The functions of ocu-miR-205 in regulating hair follicle development in Rex rabbits. BMC Dev. Biol. 20:8. doi: 10.1186/s12861-020-00213-5

Liu, Y. X. (2019). Molecular Mechanism of circna3669 as Cerna in Regulating Apoptosis of Endometrial Epithelial Cells in Dairy Goats. Xianyang: Northwest agricultural and Forestry University.

Liu, Z. H., Xiao, H. M., Li, H. P., Zhao, Y. H., Lai, S. Y., Yu, X. L., et al. (2012). Identification of conserved and novel microRNAs in cashmere goat skin by deep sequencing[J]. PLoS One 7:e50001. doi: 10.1371/journal.pone.0050001

Lv, X. Y., Gao, W., Jin, C. Y., Wang, L. H., Wang, Y., Chen, W. H., et al. (2019). Preliminary study on microR-148a and microR-10a in dermal papilla cells of Hu sheep[J]. BMC Genet. 20:70. doi: 10.1186/s12863-019-0770-8

Ma, T., Li, J. Y., Li, J. P., Wu, S. F., Xiang, B., Jiang, H. Z., et al. (2021). Expression of miRNA-203 and its target gene in hair follicle cycle development of Cashmere goat[J]. Cell Cycle 20, 204-210. doi: 10.1080/15384101.2020.1867789

McMahon, A. P., Ingham, P. W., and Tabin, C. J. (2003). Developmental roses and clinical significance of hedgehog signaling. Curr. Top. Dev. Biol. 53, 1-114. doi: 10.1016/s0070-2153(03)53002-2

Memczak, S., Jens, M., Elefsinioti, A., Torti, F., Krueger, J., and Rybak, A. (2013). Circular RNAs are a large class of animal RNAs with regulatory potency. Nature 495, 333-338. doi: 10.1038/nature11928

Milla, S. E. (2002). Molecular mechanisms regulating hair follicle development Investiga-tive. Dernatology 118, 216-225. doi: 10.1046/j.0022-202x.2001.01670. $\mathrm{x}$

Millar, S. E., Willert, K., Salinas, P. C., Roelink, H., Nusse, R., Sussman, D. J., et al. (1999). signaling in the control of hair growth and structure. Dev. Biol. 207, 133-149. doi: 10.1006/dbio.1998.9140

Mo, W. L., Jiang, J. T., Zhang, L., Lu, Q. C., Li, J., Gu, W. D., et al. (2020). Circular RNA hsa_circ_0000467 promotes the development of gastric cancer by competitively binding to MicroRNA miR-326-3p. BioMed. Res. Int. 2020:4030826.

Ogata, H., Goto, S., Sato, K., Fujibuchi, W., Bono, H., and Kanehisa, M. (1999). KEGG: kyoto encyclopedia of genes and genomes. Nucleic Acids Res. 27, 29-34.

Salmena, L., Poliseno, L., Tay, Y., Kats, L., and Pandolfi, P. P. (2011). A ceRNA hypothesis:the rosetta stone of a hidden RNA language? Cell 146, 353-358. doi: 10.1016/j.cell.2011.07.014

Schmidt-Ullrich, R., Tobin, D. J., Lenhard, D., Paus, R., and Scheidereit, C. (2006). NF-kappaB transmits Eda A1/EdaR signalling to activate Shh and cyclin D1 expression, and controls post-initiation hair placode down growth. Development 133, 1045-1057. doi: 10.1242/dev.02278

Schmittgen, T. D., and Livak, K. J. (2008). Analyzing real-time PCR data by the comparative CT method. Nat. Protoc. 3, 1101-1108. doi: 10.1038/nprot.2008.73

Shannon, P., Markiel, A., and Ozier, O. (2003). Cytoscape: a software environment for integrated models of biomolecular interaction networks. Genome Res. 13, 2498-2504. doi: 10.1101/gr.1239303

Shen, M. M., Li, T. T., Chen, F. X., Wu, P. F., Wang, Y., Chen, L., et al. (2020). Transcriptomic analysis of circRNAs and mRNAs reveals a complex regulatory network that participate in follicular development in chickens[J]. Front. Genet. 11:503. doi: 10.3389/fgene.2020.00503

Sun, W., Julie, L. Y., Huang, H. D., Shyy, J. Y. J., and Chien, S. (2010). microRNA:a master regulator of cellular processes for bioengineering systems. Ann. Rev. Biomed. Eng. 12, 1-27. doi: 10.1146/annurev-bioeng-070909-105314

Thomadakis, G., Ramoshebi, L. N., Crooks, J., Rueger, D. C., and Ripamonti, U. (1999). Immunolocalization of bone morphogenetic protein-2 and -3 and osteogenic protein-1 during murine tooth root morphogenesis and in other craniofacial structures. Eur. J. Oral. Sci. 107, 368-377. doi: 10.1046/j.0909-8836. 1999.eos107508.x

Ullrich, R., and Paus, R. (2005). Molecular principles of hair follicle induction and morphogenesis. BioEssays 27, 247-261. doi: 10.1002/bies.20184

Wang, D. Y., Chen, Z. J., Zhuang, X. N., Luo, J. Y., Chen, T., Xi, Q. Y., et al. (2020). Identifification of circRNA-associated-ceRNA networks involved in milk fat metabolism under heat stress. Int. J. Mol. Sci. 21:4162. doi: 10.3390/ ijms21114162

Wang, G. P., Guo, X. Q., Cheng, L. Y., Chu, P. P., Chen, M., Chen, Y. H., et al. (2019). An integrated analysis of the circRNA-miRNA-mRNA network reveals novel insights into potential mechanisms of cell proliferation during liver regeneration. Artif. Cells Nanomed. Biotechnol. 47, 3873-3884. doi: 10 . 1080/21691401.2019.1669623

Wang, S. H., Ge, W., Luo, Z. X., Guo, Y., Qu, L., Zhang, Z. Y., et al. (2017). Integrated analysis of coding genes and non-coding RNAs during hair follicle cycle of cashmere goat (Capra hircus). BMC Genomics 18:767. doi: 10.1186/ s12864-017-4145-0

Wei, X. F. (2017). Mechanism of miR-378a-3p, miR-107 and Related Circrna Regulating Bovine Myocyte Development. Xianyang: Northwest A \& F University.

Westholm, J. O., Miura, P., Olson, S., Shenker, S., Joseph, B., Sanfilippo, P., et al. (2014). Genome-wide analysis of drosophila circular RNAs reveals their structural and sequence properties and age-dependent neural accumulation. Cell Rep. 9, 1966-1980. doi: 10.1016/j.celrep.2014.10.062

William, R. J., and Norman, E. S. (2014). Detecting and characterizing circular RNAs. Nat. Biotechnol. 32, 453-461. doi: 10.1038/nbt.2890

Xu, Z. H., Li, P. Y., Fan, L., and Wu, M. H. (2018). The potential role of circRNA in tumor immunity regulation and immunotherapy. Front. Immunol. 22:9. doi: 10.3389/fimmu.2018.00009

Yin, R. H., Zhao, S. J., Wang, Z. Y., Zhu, Y. B., Yin, R. L., Bai, M., et al. (2020). LncRNA-599547 contributes the inductive property of dermal papilla cells in cashmere goat through miR-15b-5p/Wnt10b axis[J]. Anim. Biotechnol. 18, 1-15. doi: 10.1080/10495398.2020.1806860

You, X. T., Vlatkovic, I., Babic, I., Will, T., Epstein, I., Tushev, G., et al. (2015). Neural circular RNAs are derived from synaptic genes and regulated by development and plasticity. Nat. Neurosci. 18, 603-610. doi: 10.1038/nn.3975

Yuan, C., Wang, X. L., Geng, R. Q., He, X. L., Qu, L., and Chen, Y. L. (2013). Discovery of cashmere goat (Capra hircus) microRNAs in skin and hair follicles by Solexa sequence -ing. BMC Genomics 14:511.

Zhang, M., Ma, L., Liu, Y. H., He, Y. L., Li, G., An, X. P., et al. (2020). CircRNA006258 sponge-adsorbs miR-574-5p to regulate cell growth and milk synthesis via EVI5L in goat mammary epithelial cells.[J]. Genes (Basel) 11:718. doi: 10.3390/genes 11070718

Zhang, X. O., Wang, H. B., Zhang, Y., Lu, X. H., Chen, L. L., and Li, Y. (2014). Complementary sequence mediated exon circularization. Cell 159, 134-147. doi: 10.1016/j.cell.2014.09.001

Zhang, Y., Zhang, X. O., Tian, C., Xiang, J. F., Yin, Q. F., Xing, Y. H., et al. (2013). Circular intronic long noncoding RNAs. Mol cell 51, 792-806. doi: 10.1016/j.molcel.2013.08.017

Zhang, Y. H., Tomann, P., Andl, T., Gallant, N. M., Huelsken, J., Jerchow, B., et al. (2009). Reciprocal requirements for EDA/EDAR/NF-kappa B and Wnt/bcatenin signaling pathways in hair follicle induction. Dev Cell. 17, 49-61. doi: 10.1016/j.devcel.2009.05.011

Zhang, Y. J., Yin, J., Li, C. Q., and Li, J. Q. (2006). Study on the occurrence and development of hair follicles in fetal period of inner mongolia albas cashmere goat. J. Anim. Husbandry Vet. Med. 37, 761-768.

Zhang, Y. J., Yin, J., Li, J. Q., and Li, C. Q. (2007). Study on the structure and morphogenesis of cashmere follicles in Albas cashmere goats, Inner Mongolia. Chin. J. Agric. Sci. 40, 1017-1023.

Zhang, Z. Z. (2018). Molecular Mechanism of miR-103 Regulating Adipose Development by Binding circRNAs. Xianyang: Northwest A \& F University.

Zhao, B. H., Chen, Y., Hu, S. S., Yang, N. S., Wang, M. M., Liu, M., et al. (2019). Systematic analysis of non-coding RNAs involved in the angora rabbit (Oryctolagus cuniculus) hair follicle cycle by RNA sequencing. Front. Genet. 10:407. doi: 10.3389/fgene.2019.00407

Zhao, J., Yang, X. W., Li, J. T., Wang, Q., Wang, L., and Wang, G. T. (2019). Construction and functional enrichment analysis of circrna miRNA mRNA regulatory network in hepatocellular carcinoma based on high-throughput sequencing. Jo. Clin. Hepatobiliary Dis. 35, 1740-1744.

Zheng, Y. Y., Wang, Z. Y., Zhu, Y. B., Wang, W., Bai, M., Jiao, Q., et al. (2019). LncRNA-000133 from secondary hair follicle of Cashmere goat: identification, regulatory network and its effects on inductive property of dermal papilla cells. Anim. Biotechnol. 31, 122-134. doi: 10.1080/10495398.2018.1553788

Zhou, G. X. (2018). Screening of Key Genes of Secondary Hair Follicle cycle Cycle in Northern Shaanxi White Cashmere Goat and Functional Verification of Lhx2 and miR-144. Xianyang: Northwest A \& F University.

Zhou, G. X., Kang, D. J., Ma, S., Wang, X. T., Gao, Y., Yang, Y. X., et al. (2018). Integrative analysis reveal sncRNA-mediated molecular 
regulatory network driving secondary hair follicle regression in cashmere goats. BMC Genomics 19:222. doi: 10.1186/s12864-0184603-3

Zhou, X. B. (2016). Optimization of Culture Medium Composition of Secondary Dermal Papilla Cells of Shanbei White Cashmere Goat and the effect of miR-206 on it Regulation research. Xianyang: Northwest A \& F University.

Zong, Z. H., Liu, Y., Chen, S., and Zhao, Y. (2020). Circ_PUM1 promotes the development of endometrial cancer by targeting the miR-136/NOTCH3 pathway. J. Cell. Mol. Med. 24, 4127-4135. doi: 10.1111/jcmm.15 069
Conflict of Interest: The authors declare that the research was conducted in the absence of any commercial or financial relationships that could be construed as a potential conflict of interest.

Copyright (c) 2021 Shang, Wang, Ma, Di, Wu, Hai, Rong, Pan, Liang, Wang, Wang, Liu, Zhao, Wang, Li and Zhang. This is an open-access article distributed under the terms of the Creative Commons Attribution License (CC BY). The use, distribution or reproduction in other forums is permitted, provided the original author(s) and the copyright owner(s) are credited and that the original publication in this journal is cited, in accordance with accepted academic practice. No use, distribution or reproduction is permitted which does not comply with these terms. 